\title{
Correction to: Sources of Uncertainty in the Tropical Pacific Warming Pattern under Global Warming Projected by Coupled Ocean-Atmosphere Models
}

\section{Correction to:}

J. Ying, Sources of Uncertainty in the Tropical Pacific Warming Pattern under Global Warming Projected by Coupled Ocean-Atmosphere Models, Springer Theses, https://doi.org/10.1007/978-981-32-9844-6

In the original version of the book, the following belated corrections have been incorporated:

The original version of the book was inadvertently published without the second affiliation "Southern Marine Science and Engineering Guangdong Laboratory (Zhuhai)" of the author "Jun Ying" in frontmatter, which has now been included.

The organization name "Southern Marine Science and Engineering Guangdong Laboratory (Zhuhai)" was missed in the acknowledgement. This has now been updated.

The erratum book has been updated with the change.

The updated version of the book can be found at https://doi.org/10.1007/978-981-32-9844-6 